\title{
OPEN Association between pet ownership and sleep in the Swedish CArdioPulmonary biolmage Study (SCAPIS)
}

\author{
Lieve T. van Egmond ${ }^{1 \bowtie}$, Olga E. Titova ${ }^{2}$, Eva Lindberg ${ }^{3}$, Tove Fall ${ }^{4}$ \& Christian Benedict ${ }^{1}$
}

Preliminary findings suggest that pets may impact the owner's sleep. By using data from the Swedish CArdioPulmonary bloimage Study (SCAPIS) cohort, we aimed to investigate the association of pet ownership with the following self-reported sleep outcomes in 3788 to 4574 participants: (i) achieving the recommended daily sleep duration for adults (i.e., at least $7 \mathrm{~h}$ per day); (ii) sleep quality as measured by the Pittsburgh Sleep Quality Index (a score of $>5$ indicating poor sleep quality); and (iii) difficulty falling or staying asleep. Sleep metrics were not associated with pet ownership, dog ownership, and dog walking when controlling the logistic regression for possible confounders (e.g., shift work, lack of social interaction, and chronic stress). In contrast, cat ownership was associated with a higher odds ratio of failing to achieve the recommended duration of $7 \mathrm{~h}$ of sleep per day (adjusted odds ratio $[95 \% \mathrm{CI}]: 1.18[1.02,1.37]$ versus non-cat owners). Our findings suggest that certain pet groups might have a more significant impact on the owner's sleep than others. As the observed association between cat ownership and short sleep duration might be a chance finding, this observation should be seen as hypothesis-generating only.

The potential therapeutic value of pets to improve sleep has been the subject of recent research. The most extensive study to date, a cross-sectional examination of 6,575 participants of the Whitehall II study aged between 59 and 79 , revealed that pet owners had less trouble falling asleep than non-pet owners ${ }^{1}$. Companionship, security, physical activity (e.g., due to dog walking), and relaxation may represent possible mechanisms through which pets facilitate the owners' ability to fall asleep. On the other hand, in the same cohort, pet ownership was also associated with feeling more tired after waking up ${ }^{1}$, possibly because pet owners may be waking due to their pets. The results of smaller studies painted a similarly complex picture of the association between pet ownership and sleep. A questionnaire study involving 801 subjects (ages ranged from 20 to 50 years) found that men who owned a pet reported better subjective sleep quality ${ }^{2}$. Using accelerometry to measure sleep objectively for seven nights, a separate study involving 40 healthy dog owners without sleep disorders (mean age 44 years) found that sleep efficiency was lower if the dog was on the bed instead of merely in the room ${ }^{3}$. Extending this finding, another study involving 150 patients (age range not specified) found that about $20 \%$ of the pet owners described their pets as disruptive ${ }^{4}$. The heterogeneity of the results and the fact that this topic is not well-researched highlights that more studies are required.

In the present study, using survey data from the Swedish CArdioPulmonary bioImage Study (SCAPIS), we aimed to examine the following hypotheses: (i) pet owners have higher odds of achieving the recommended daily sleep duration for adults (i.e., at least $7 \mathrm{~h}$ per day); (ii) pet owners report better sleep quality as measured by the Pittsburgh Sleep Quality Index (PSQI; see ref.5); and (iii) pet owners have fewer complaints of difficulty falling or staying asleep when compared to non-pet owners. We also investigated if these possible associations exist among dog or cat owners.

As regular physical activity has been shown to improve various sleep parameters, e.g., sleep onset latency and sleep quality ${ }^{6-8}$, we also investigated whether dog owners who reported to be mainly responsible for dog walking would exhibit better sleep.

${ }^{1}$ Department of Neuroscience, Uppsala University, Husargatan 3, Box 593, 75124 Uppsala, Sweden. ${ }^{2}$ Department of Surgical Sciences, Unit of Medical Epidemiology, Uppsala University, Uppsala, Sweden. ${ }^{3}$ Department of Medical Sciences, Respiratory, Allergy and Sleep Research, Uppsala University, Uppsala, Sweden. ${ }^{4}$ Department of Medical Sciences, Molecular Epidemiology and Science for Life Laboratory, Uppsala University, Uppsala, Sweden. ${ }^{\bowtie}$ email: lieve.van.egmond@neuro.uu.se 


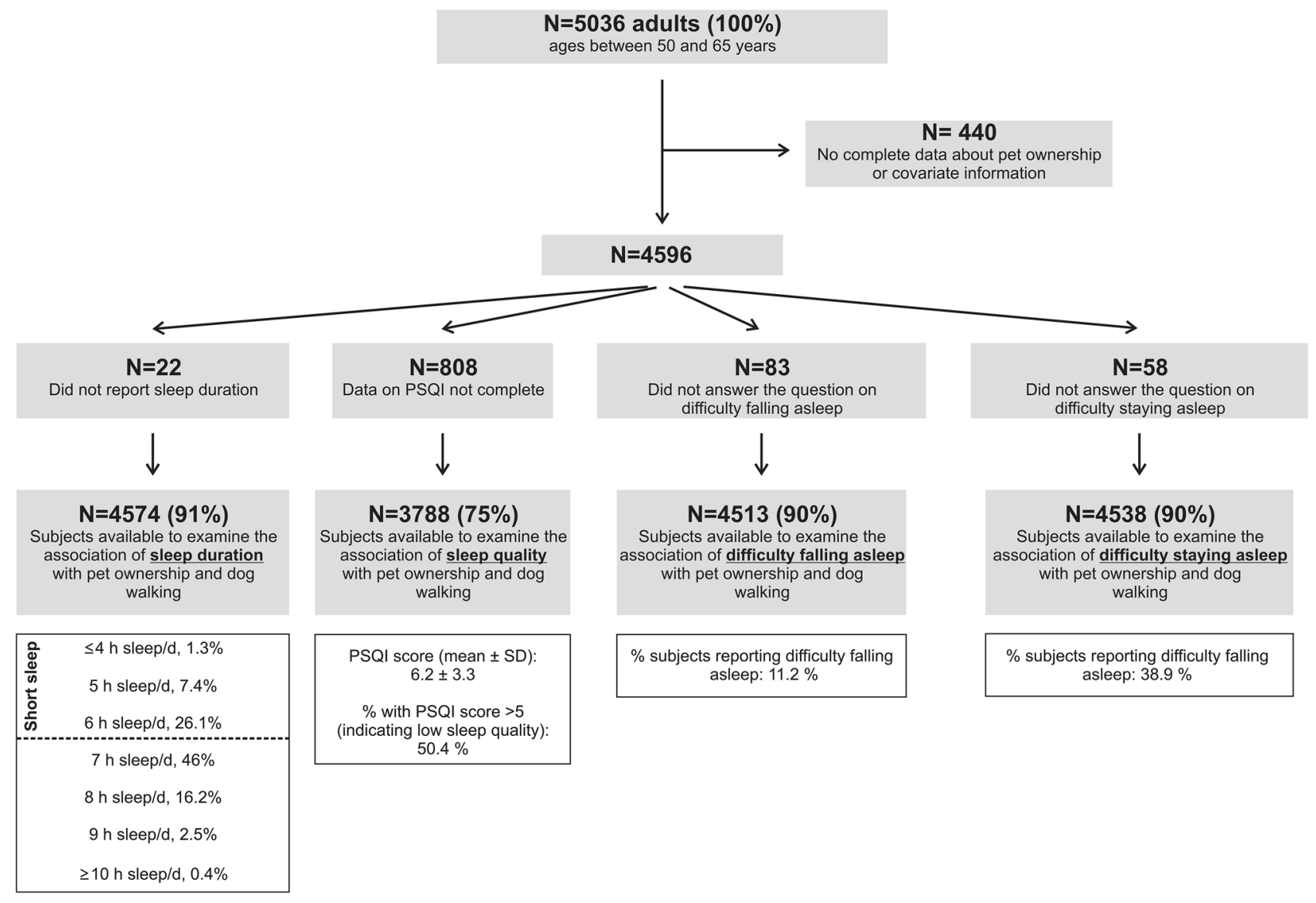

Figure 1. Flowchart.

\section{Material and methods}

Population and study design. The SCAPIS study is a Swedish nationwide population-based cohort mainly designed to research cardiovascular and chronic obstructive pulmonary diseases. Between 2013 and 2018, 30,154 men and women aged 50 to 65 were recruited from a random population sample by six Swedish university hospitals (Gothenburg, Linköping, Malmö/Lund, Stockholm, Umeå, and Uppsala). The primary aim of SCAPIS is to improve the risk prediction of cardiopulmonary diseases and optimize the ability to study disease mechanisms ${ }^{9}$.

In addition to surveying the general health status and lifestyle habits, all Uppsala participants (but not the other areas) were also asked about pet ownership (see below). Following exclusions (detailed in Fig. 1), data from 4,574 subjects from the Uppsala cohort were available to examine the association between pet ownership and sleep duration, 3788 subjects for the sleep quality analysis, 4513 subjects for the difficulty falling asleep analysis, and 4538 subjects for the difficulty staying asleep analysis, respectively. The Regional Ethical Review Board in Uppsala (Etikprövningsmyndigheten Uppsala; approval number DNR2019-05343) approved the study, and all participants provided written informed consent. All investigations were conducted in accordance with the Declaration of Helsinki.

Exposure-pet ownership. Pet ownership was assessed by the question: "Do you have a pet in the household?". Participants could answer with "yes", "no", or "I can't or don't want to answer". The answer "I can't or don't want to answer" was treated as missing data. If answering yes to the pet ownership question, participants could fill in which and how many pets they had, divided into "dog", "cat", "rabbit", "bird", or "other". Dog owners were also asked about their dog walking engagement. Possible answer options were: "I mainly take care of the dog's daily exercise", "I take care of about half of the dog's daily exercise", "I take care of a small part of the dog's daily exercise", "I have no dog", and "I can't or don't want to answer". Dog owners who chose the answer options "I have no dog" or "I can't or don't want to answer" were treated as non-dog owners in the analysis regarding the association between dog walking and sleep.

Outcome-sleep. Participants were asked, "How many hours do you normally sleep during a day" with the following answer options: " 4 h or less per day"; "5 h per day"; "6 h per day"; "7 h per day"; "8 h per day"; "9 h per day"; and "10 or more hours per day". In the present analysis, " $7 h$ " or more were classified as achieving the recommended daily sleep duration. Note that this question was part of the SCAPIS core questionnaire, i.e., it did 
not derive from the PSQI where the respondent is typically asked several questions regarding sleep, including a question on how many hours (s)he habitually slept during the past month.

Participants' sleep quality was measured using the PSQI ${ }^{5}$. As described elsewhere, the PSQI surveys various sleep characteristics during the past month, such as sleep latency (i.e., how long it takes to fall asleep). Each item is scored on a $0-3$ interval scale $(0=$ not during the past month; $1=$ Less than once a week; $2=$ once or twice a week; and $3=$ Three or more times a week). The answer "Three or more times a week" to these questions was classified as having this sleep problem. Overall, a score of $>5$ defines poor sleep quality ${ }^{5}$.

In SCAPIS, participants could also choose the answer option "I can't or don't want to answer" when answering the PSQI components. This answer option does typically not exist in the PSQI ${ }^{5}$. To ensure that our results can be compared with past and future investigations using the PSQI, participants who answered any questions of the PSQI with "I can't or don't want to answer" were excluded from the analysis.

The PSQI questions "During the past month, how often have you had trouble sleeping because you cannot get to sleep within 30 min?" and "During the past month, how often have you had trouble sleeping because you wake up in the middle of the night or early morning?" were used in a separate analysis to evaluate whether pet ownership associated with difficulty falling and difficulty staying asleep, respectively. The answer "Three or more times a week" to these questions was classified as having this sleep problem.

Confounders. We applied d-separation criteria on directed acyclic graphs (DAGs; see ref. ${ }^{10}$ ) to identify potential confounders to include in the regression model (see Supplementary Figure S1). DAGs is a widely used method to depict graphically assumed causal relationships between predictor, outcome, and confounder variables.

For the analysis, education was divided into university degree vs no university degree. A high level of social interaction was defined as meeting more than two close friends every week. Physical activity status was divided into $\geq 2 \mathrm{~h}$ of modest to heavy exercise/week vs $<2 \mathrm{~h}$ of modest to heavy exercise/week. Alcohol consumption frequency was divided into $\geq 2$ times/week vs $<2$ times per week. The following question assessed socio-economic stability: "Could you raise 20,000 SEK within a week if required?" (answer options: yes, no). Furthermore, subjects reported whether they work night shifts, have been diagnosed with sleep apnea, experienced a high-stress load during the past 12 months (yes, no), and if they currently smoke. Some questions also had the response option "I can't or don't want to answer". Hence, these confounders were entered as variables with three answer options into the analysis. Diabetes was either confirmed by questionnaire (yes, no) or when participants without previously known diabetes had a blood glucose level of at least $7 \mathrm{mmol} / \mathrm{l}$ or an $\mathrm{HbA1c}$ level of at least $48 \mathrm{mmol} / \mathrm{mol}$. The weight and height were measured during the physical SCAPIS examination and used to calculate BMI. Age was recorded at the time of the examination.

Statistical analysis. All analyses were performed using IBM SPSS Statistics 24 (SPSS Inc. Chicago, IL, USA). Logistic regression was used to investigate the association of pet ownership (any pet, dogs, or cats) and dog walking with sleep. Results from adjusted (including all confounders described above) logistic regressions are reported. Overall, a $P$ value $<0.05$ was considered significant.

\section{Results}

Cohort characteristics. As shown in Table 1, all subsamples used in the present analysis had a nearly balanced gender distribution. The vast majority of participants reported a stable socio-economic status, about half had a university degree, and slightly more than $10 \%$ stated that they were less socially interactive. For more cohort characteristics, see Table 1.

The population median sleep duration was $7 \mathrm{~h} /$ night, and $34.8 \%$ of the sleep duration subsample participants reported that they habitually slept less than $7 \mathrm{~h}$ per day. $50.4 \%$ reported low sleep quality (i.e., PSQI score $>5$ ) in the PSQI cohort. In the subsample to investigate the association between trouble falling asleep and pet ownership/dog walking, $11.2 \%$ reported difficulty falling asleep. Finally, $38.9 \%$ of the middle-aged to older subjects complained about difficulty staying asleep.

Association between pet ownership and sleep. The adjusted logistic regression analyses revealed no significant associations between pet ownership (any pet vs no pet) and sleep outcomes. Similar null-results were observed when comparing dog owners with non-dog owners. In contrast, we found that cat owners have a higher odds ratio of failing to achieve the recommended duration of $7 \mathrm{~h}$ of sleep per day $(1.18[1.02,1.37]$ vs noncat owners, $P=0.028$ ). No cat ownership associations with sleep quality, difficulty falling asleep, and difficulty staying asleep were found. Please see Table 2.

Another aim of the present study was to investigate the association of dog walking engagement with sleep. When splitting dog owners into subgroups based on dog walking engagement, no significant associations with the sleep outcomes emerged (Table 3 ).

Overall, no significant interactions between pet ownership and sex were found for any of the sleep outcomes (pet ownership ${ }^{\star}$ sex: $P \geq 0.14$; dog ownership $^{\star}$ sex: $P \geq 0.42$; cat ownership ${ }^{\star}$ sex: $P \geq 0.46$; and $\operatorname{dog}$ walking ${ }^{\star}$ sex: $P \geq 0.23$ ). Note that similar null-results for the association between the exposure and outcome variables were found when using unadjusted logistic regression analyses, except for a significant association between dog walking and difficulty staying asleep $(1.33[1.04,1.71]$ compared to non-dog owners, $P=0.025)$. 


\begin{tabular}{|c|c|c|c|c|}
\hline & \multicolumn{4}{|c|}{ SCAPIS subsamples } \\
\hline & Sleep duration & PSQI & DIS & DSA \\
\hline Number of subjects (n) & 4574 & 3788 & 4513 & 4538 \\
\hline Age (years) & $57.7 \pm 4.4$ & $57.5 \pm 4.4$ & $57.7 \pm 4.4$ & $57.6 \pm 4.4$ \\
\hline BMI $\left(\mathrm{kg} / \mathrm{m}^{2}\right)$ & $27.0 \pm 4.4$ & $26.7 \pm 4.2$ & $27.0 \pm 4.4$ & $27.0 \pm 4.4$ \\
\hline \multicolumn{5}{|l|}{ Sex } \\
\hline Men & $2207(48.3)$ & $1856(49.0)$ & $2172(48.1)$ & $2187(48.2)$ \\
\hline Women & $2367(51.7)$ & $1932(51.0)$ & $2341(51.9)$ & $2351(51.8)$ \\
\hline \multicolumn{5}{|l|}{ Nightshift work } \\
\hline No & $4117(90.0)$ & $3456(91.2)$ & $4065(90.1)$ & $4088(90.1)$ \\
\hline Yes & $330(7.2)$ & $241(6.4)$ & $323(7.2)$ & $324(7.1)$ \\
\hline Don't know/don't want to report & $127(2.8)$ & $91(2.4)$ & $125(2.8)$ & $126(2.8)$ \\
\hline \multicolumn{5}{|l|}{ Stable socio-economic status } \\
\hline No & $246(5.4)$ & $157(4.1)$ & $242(5.4)$ & $248(5.5)$ \\
\hline Yes & $4245(92.8)$ & $3591(94.8)$ & $4190(92.8)$ & $4211(92.8)$ \\
\hline Don't know/don't want to report & $83(1.8)$ & $40(1.1)$ & $81(1.8)$ & $79(1.7)$ \\
\hline \multicolumn{5}{|l|}{ Sleep apnea diagnosis } \\
\hline No & $4368(95.5)$ & $3636(96.0)$ & $4315(95.6)$ & $4333(95.5)$ \\
\hline Yes & $177(3.9)$ & $137(3.6)$ & $173(3.8)$ & $176(3.9)$ \\
\hline Don't know/don't want to report & $29(0.6)$ & $15(0.4)$ & $25(0.6)$ & $29(0.6)$ \\
\hline \multicolumn{5}{|l|}{ Diabetes diagnosis } \\
\hline No & $4201(91.8)$ & $3506(92.6)$ & 4149 (91.9) & $4170(91.9)$ \\
\hline Yes & $373(8.2)$ & $282(7.4)$ & $364(8.1)$ & $368(8.1)$ \\
\hline \multicolumn{5}{|l|}{ Current smoker } \\
\hline No & $4109(89.8)$ & $3440(90.8)$ & 4059 (89.9) & $4077(89.8)$ \\
\hline Yes & $405(8.9)$ & $301(7.9)$ & $395(8.8)$ & $401(8.8)$ \\
\hline Don't know/don't want to report & $60(1.3)$ & $47(1.2)$ & $59(1.3)$ & $60(1.3)$ \\
\hline \multicolumn{5}{|c|}{ Perceived stress level in the past year } \\
\hline Low & $3580(78.3)$ & $3021(79.8)$ & $3532(78.3)$ & $3549(78.2)$ \\
\hline High & $964(21.1)$ & $760(20.1)$ & $950(21.1)$ & $957(21.1)$ \\
\hline Don't know/don't want to report & $30(0.7)$ & $7(0.2)$ & $31(0.7)$ & $32(0.7)$ \\
\hline \multicolumn{5}{|l|}{ Social interaction } \\
\hline Low & $591(12.9)$ & $432(11.4)$ & $585(13.0)$ & $588(13.0)$ \\
\hline High (>2 people/week) & $3949(86.3)$ & $3338(88.1)$ & $3898(86.4)$ & $3918(86.3)$ \\
\hline Don't know/don't want to report & $34(0.7)$ & $18(0.5)$ & $30(0.7)$ & $32(0.7)$ \\
\hline \multicolumn{5}{|l|}{ Alcohol consumption frequency } \\
\hline Low & $2882(63.0)$ & $2306(60.9)$ & $2837(62.9)$ & $2855(62.9)$ \\
\hline High ( $\geq 2$ times/week) & $1677(36.7)$ & $1472(38.9)$ & $1661(36.8)$ & $1667(36.7)$ \\
\hline Don't know/don't want to report & $15(0.3)$ & $10(0.3)$ & $15(0.3)$ & $16(0.4)$ \\
\hline \multicolumn{5}{|l|}{ Physical activity } \\
\hline Never/seldom & $2517(55.0)$ & $2017(53.2$ & $2480(55.0)$ & $2497(55.0)$ \\
\hline Regular ( $\geq 2$ h/week) & $1989(43.5)$ & $1738(45.9)$ & $1969(43.6)$ & $1972(43.5)$ \\
\hline Don't know/don't want to report & $68(1.5)$ & $33(0.9)$ & $64(1.4)$ & $69(1.5)$ \\
\hline \multicolumn{5}{|l|}{ Educational status } \\
\hline No university degree & $2222(48.6)$ & $1763(46.5)$ & $2183(48.4)$ & $2200(48.5)$ \\
\hline University degree & $2342(51.2)$ & $2023(53.4)$ & $2320(51.4)$ & $2329(51.3)$ \\
\hline Don't know/don't want to report & $10(0.2)$ & $2(0.1)$ & $10(0.2)$ & $9(0.2)$ \\
\hline
\end{tabular}

Table 1. Cohort characteristics. All values reported as n (\% group) unless otherwise specified. PSQI, Pittsburgh Sleep Quality Index; n, number; DIS, difficulty initiating sleep; DSA, difficulty staying asleep; DSA; difficulty staying asleep; BMI, body mass index.

\section{Discussion}

In the present study, we found a significant association between cat ownership and not reaching $7 \mathrm{~h}$ of sleep per night, as recommended for adults. In contrast, owning a dog, the second most prevalent pet in our cohort, was not associated with sleep. We neither found an association between general pet ownership and sleep. These findings might suggest that certain pet groups have a greater impact on the owner's sleep than others. 


\begin{tabular}{|c|c|c|c|c|c|c|}
\hline & \multirow{2}{*}{\begin{tabular}{|l|} 
Pet owner \\
OR $[95 \% \mathrm{CI}], P$ \\
\end{tabular}} & \multirow[b]{2}{*}{ Non-pet owner } & \multirow{2}{*}{$\begin{array}{l}\text { Cat owner } \\
\text { OR }[95 \% \mathrm{CI}], P\end{array}$} & \multirow[b]{2}{*}{ Non-cat owner } & \multirow{2}{*}{\begin{tabular}{|l|} 
Dog owner \\
OR $[95 \% \mathrm{CI}], P$ \\
\end{tabular}} & \multirow[b]{2}{*}{ Non-dog owner } \\
\hline & & & & & & \\
\hline \multicolumn{7}{|c|}{ Odds of experiencing poor sleep quality (PSQI $>5$ ) } \\
\hline No. of subjects & 1406 & 2382 & 863 & 2925 & 613 & 3175 \\
\hline PSQI >5 (\%) & 51.7 & 49.6 & 53.3 & 49.5 & 50.6 & 50.4 \\
\hline Fully adjusted ${ }^{a}$ & $\begin{array}{l}1.00[0.87,1.15] \\
P=0.997\end{array}$ & 1 & $\begin{array}{l}1.11[0.94,1.30] \\
P=0.22\end{array}$ & 1 & $\begin{array}{l}0.92[0.76,1.10] \\
P=0.35\end{array}$ & 1 \\
\hline \multicolumn{7}{|c|}{ Odds of having difficulty with initiating sleep at night } \\
\hline No. of subjects & 1648 & 2865 & 1011 & 3502 & 728 & 3785 \\
\hline DIS (\%) & 11.4 & 11.1 & 12.5 & 10.8 & 11.1 & 11.2 \\
\hline Fully adjusted ${ }^{a}$ & $\begin{array}{l}0.93[0.76,1.14] \\
P=0.51\end{array}$ & 1 & $\begin{array}{l}1.08[0.86,1.35] \\
P=0.52\end{array}$ & 1 & $\begin{array}{l}0.89[0.68,1.16] \\
P=0.38\end{array}$ & 1 \\
\hline \multicolumn{7}{|c|}{ Odds of having difficulty with staying asleep at night } \\
\hline No. of subjects & 1657 & 2881 & 1017 & 3521 & 736 & 3802 \\
\hline DSA (\%) & 39.6 & 38.5 & 40.4 & 38.5 & 40.2 & 38.7 \\
\hline Fully adjusted ${ }^{a}$ & $\begin{array}{l}1.01[0.89,1.15] \\
P=0.84\end{array}$ & 1 & $\begin{array}{l}1.05[0.91,1.22] \\
P=0.49\end{array}$ & 1 & $\begin{array}{l}1.01[0.86,1.20] \\
P=0.89\end{array}$ & 1 \\
\hline \multicolumn{7}{|c|}{ Odds of sleeping $<7 \mathrm{~h}$ per day } \\
\hline No. of subjects & 1669 & 2905 & 1026 & 3548 & 741 & 3833 \\
\hline$<7$ h/day $(\%)$ & 36.1 & 34.0 & 38.6 & 33.7 & 35.4 & 34.7 \\
\hline Fully adjusted ${ }^{a}$ & $\begin{array}{l}1.04[0.91,1.18] \\
P=0.60\end{array}$ & 1 & $\begin{array}{l}1.18[1.02,1.37] \\
P=0.028\end{array}$ & 1 & $\begin{array}{l}0.96[0.81,1.13] \\
P=0.59\end{array}$ & 1 \\
\hline
\end{tabular}

Table 2. Association between pet ownership and sleep in the SCAPIS cohort (Uppsala). CI, confidence interval; DIS, difficulty initiating sleep; DSA, difficulty staying asleep; No., number; OR, odds ratio; PSQI, Pittsburgh Sleep Quality Index. a Logistic regression was adjusted for the following variables: sex, education, age, BMI, social interaction, regular physical activity, alcohol frequency, socio-economic stability, night shift work, diabetes, sleep apnea, constant stress, current smoking.

\begin{tabular}{|c|c|c|c|c|}
\hline & Majority of dog walking & About half of dog walking & Lesser part of dog walking & \multirow[b]{2}{*}{ Non-dog owners } \\
\hline & OR $[95 \% \mathrm{CI}], P$ & OR $[95 \% \mathrm{CI}], P$ & OR $[95 \% \mathrm{CI}], P$ & \\
\hline \multicolumn{5}{|c|}{ Odds of experiencing poor sleep quality (i.e., PSQI $>5$ ) } \\
\hline No. of subjects & 217 & 198 & 182 & 3175 \\
\hline PSQI > 5 (\% group) & 49.3 & 52.5 & 48.4 & 50.4 \\
\hline Fully adjusted $^{\mathrm{a}}$ & $0.81[0.61,1.09], P=0.17$ & $1.06[0.78,1.43], P=0.71$ & $0.86[0.63,1.18], P=0.34$ & 1 \\
\hline \multicolumn{5}{|c|}{ Odds of having difficulty with initiating sleep at night } \\
\hline No. of subjects & 261 & 228 & 217 & 3785 \\
\hline DIS (\% group) & 12.3 & 10.5 & 10.6 & 11.2 \\
\hline Fully adjusted $^{\mathrm{a}}$ & $0.93[0.62,1.40], P=0.74$ & $0.90[0.57,1.42], P=0.74$ & $0.86[0.54,1.37], P=0.53$ & 1 \\
\hline \multicolumn{5}{|c|}{ Odds of having difficulty with staying asleep at night } \\
\hline No. of subjects & 265 & 228 & 221 & 3802 \\
\hline DSA (\% group) & 45.7 & 40.8 & 33.5 & 38.7 \\
\hline Fully adjusted $^{\mathrm{a}}$ & $1.18[0.92,1.53], P=0.20$ & $1.09[0.83,1.45], P=0.53$ & $0.79[0.58,1.06], P=0.11$ & 1 \\
\hline \multicolumn{5}{|c|}{ Odds of sleeping $<7 \mathrm{~h}$ per day } \\
\hline No. of subjects & 265 & 229 & 224 & 3833 \\
\hline$<7$ h/d (\% group) & 35.1 & 33.2 & 36.2 & 34.7 \\
\hline Fully adjusted $^{\mathrm{a}}$ & $0.94[0.72,1.23], P=0.65$ & $0.90[0.68,1.20], P=0.47$ & $0.97[0.73,1.29], P=0.82$ & 1 \\
\hline
\end{tabular}

Table 3. Logistic regression analysis to investigate the association between dog walking and sleep in the SCAPIS cohort (Uppsala). CI, confidence interval; DIS, difficulty initiating sleep; DSA, difficulty staying asleep;

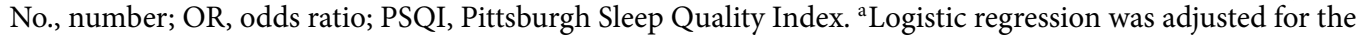
following variables: sex, education, age, BMI, social interaction, regular physical activity, alcohol frequency, socio-economic stability, night shift work, diabetes, sleep apnea, constant stress, current smoking.

Our results did not show any association between general pet ownership and sleep. They contrast with previous findings from the Whitehall II study, where pet ownership was associated with less trouble falling asleep but less refreshed awakening ${ }^{1}$. Possible explanations for these contrasting results could relate to differences in sample size, age range, how subjective sleep was measured, and adjustments of confounders. 
Our findings do not suggest that general pet ownership alters sleep. However, it must be kept in mind that pets may, under certain circumstances, still improve the owner's sleep, e.g., among people suffering from anxiety, depression, loneliness, and grief. For instance, in a study involving 340 older adults from China, it was demonstrated that pet engagement eased depression ${ }^{11}$, which can cause sleep problems ${ }^{12}$.

It is also possible that pet ownership can impair sleep. For instance, in a previous study, one-fifth of the pet owners reported that their co-sleeping pet disrupted their sleep ${ }^{4}$. Extending these findings, we found that cat owners exhibited an increased risk of sleeping too short. Cats can display crepuscular behaviour, i.e., they are primarily active at dawn and dusk. Consequently, when co-sleeping with a cat, the owner's sleep might become disrupted.

Considering that owning a dog might be particularly beneficial to sleep, e.g., due to increased physical activity and spending time outside, we also investigated if there is a link between dog ownership and dog walking engagement with sleep. Contrary to our expectations, we found no associations when controlling for confounders known to affect sleep, such as chronic stress, shift work, and lack of social interaction. We did not have information about the breed, age, and pedigree of dogs, which may moderate the association between dog ownership and health outcomes, including sleep. For instance, owning a dog of mixed pedigree or a dog belonging to the 'companion/toy' breed group was associated with hypertension and dyslipidemia in the dog owner ${ }^{13}$. Additionally, owning a dog from the 'Spitz/primitive' breed and the combined group of' active dog breeds' was associated with a lower risk of diabetes mellitus ${ }^{13}$. Altogether, our study does not provide compelling evidence for the hypothesis that dog walking may result in overall better sleep.

Several strengths and limitations apply to our study. The primary strength of the present study is its large sample size. Moreover, results were robust to adjustments for multiple potential confounders, such as participants' age and educational level, yet a possibility of residual confounding remains. Another strength is that the $\mathrm{PSQI}^{5}$, a widely used questionnaire to assess a person's sleep quality, was used in the present analysis. On the other hand, the accuracy of self-reported sleep length might be subject to recall bias. Another limitation of the current study is the restricted age range (50-65), limiting the generalisability of the results. Besides, we have no information regarding pet engagement (except dog walking). For instance, co-sleeping with pets can be perceived as disruptive to sleep ${ }^{4}$. In SCAPIS, it was not surveyed either whether non-pet owners may have regular contact with visiting pets. A study involving 100 residents from nursing homes (ages ranged between 79 and 90 years) showed that their sleep duration was increased when a dog accompanied visitors ${ }^{14}$. When interpreting our results, it must be borne in mind that the observed association between cat ownership and short sleep duration might be a chance finding. Thus, this observation should be seen as hypothesis-generating only. Finally, although our findings show an association between cat ownership and short sleep duration, these observational results do not imply a causal relationship.

\section{Conclusions}

We found that owning a cat, the most prevalent pet group in the present study, was associated with increased odds of sleeping less than the recommended seven hours per day. Whether this means that cats represent a risk factor for short sleep duration cannot be derived from the present observational study. Therefore, future studies should more thoroughly investigate the various aspects of cat ownership, e.g., the cat's breed, age, and co-sleeping with the cat.

Received: 28 November 2020; Accepted: 22 March 2021

Published online: 02 April 2021

\section{References}

1. Mein, G. \& Grant, R. A cross-sectional exploratory analysis between pet ownership, sleep, exercise, health and neighbourhood perceptions: The Whitehall II cohort study. BMC Geriatr. 18, 176 (2018).

2. Defelipe, R., Savalli, C. \& Otta, E. Demographics and self-reported well-being of Brazilian adults as a function of pet ownership: A pilot study. Heliyon 6, e04069. https://doi.org/10.1016/j.heliyon.2020.e04069 (2020).

3. Patel, S. I. et al. The effect of dogs on human sleep in the home sleep environment. Mayo Clin. Proc. 92, 1368-1372 (2017).

4. Krahn, L. E., Tovar, M. D. \& Miller, B. Are pets in the bedroom a problem?. Mayo Clin. Proc. 90, 1663-1665 (2015).

5. Buysse, D. J., Reynolds, C. F., Monk, T. H., Berman, S. R. \& Kupfer, D. J. The Pittsburgh sleep quality index: A new instrument for psychiatric practice and research. Psychiatry Res. 28, 193-213 (1989).

6. Tan, X., van Egmond, L. T., Cedernaes, J. \& Benedict, C. The role of exercise-induced peripheral factors in sleep regulation. Mol. Metab. 42, 101096. https://doi.org/10.1016/j.molmet.2020.101096 (2020).

7. Jurado-Fasoli, L. et al. Exercise training improves sleep quality: A randomized controlled trial. Eur. J. Clin. Invest. 50, e13202. https://doi.org/10.1111/eci.13202 (2020).

8. Tan, X., Alén, M., Wiklund, P., Partinen, M. \& Cheng, S. Effects of aerobic exercise on home-based sleep among overweight and obese men with chronic insomnia symptoms: a randomized controlled trial. Sleep Med. 25, 113-121 (2016).

9. Bergström, G. et al. The Swedish CArdioPulmonary BioImage Study: Objectives and design. J. Intern. Med. 278, 645-659 (2015).

10. Textor, J., Hardt, J. \& Knüppel, S. DAGitty: A graphical tool for analyzing causal diagrams. Epidemiology 22, 745 (2011).

11. Cheung, C. \& Kam, P. K. Conditions for pets to prevent depression in older adults. Aging Ment. Heal. 22, 1627-1633 (2018).

12. Pandi-Perumal, S. R. et al. Clarifying the role of sleep in depression: A narrative review. Psychiatry Res. 291, 113239. https://doi. org/10.1016/j.psychres.2020.113239 (2020).

13. Mubanga, M. et al. Dog ownership and cardiovascular risk factors: a nationwide prospective register-based cohort study. $B M J$ Open 9, e023447. https://doi.org/10.1136/bmjopen-2018-023447 (2019).

14. Thodberg, K. et al. Therapeutic effects of dog visits in nursing homes for the elderly. Psychogeriatrics 16, 289-297 (2016). 


\section{Author contributions}

Conceptualization: all authors; Data curation: L.v.E.; Formal analysis: L.v.E., C.B.; Funding acquisition: C.B.; Visualization: L.v.E.; Roles/Writing - original draft: L.v.E.; Writing - review \& editing: O.T., E.L., T.F., C.B.

\section{Funding}

Open access funding provided by Uppsala University. The authors' work is funded by the Novo Nordisk Foundation (C.B., NNF19OC0056777), Skandia (C.B.), Swedish Brain Research Foundation (C.B., FO2020-0044), and Swedish Research Council (C.B., 2015-03100).

\section{Competing interests}

L.v.E., O.E.T., E.L., and T.F. have no conflicts of interest to report. C.B. reports that he served as a paid member of a scientific advisory board for Repha $\mathrm{GmBH}$, Langenhagen, Germany. C.B. declares no other conflict of interests.

\section{Additional information}

Supplementary Information The online version contains supplementary material available at https://doi.org/ 10.1038/s41598-021-87080-7.

Correspondence and requests for materials should be addressed to L.T.v.E.

Reprints and permissions information is available at www.nature.com/reprints.

Publisher's note Springer Nature remains neutral with regard to jurisdictional claims in published maps and institutional affiliations.

(c) (i) Open Access This article is licensed under a Creative Commons Attribution 4.0 International License, which permits use, sharing, adaptation, distribution and reproduction in any medium or format, as long as you give appropriate credit to the original author(s) and the source, provide a link to the Creative Commons licence, and indicate if changes were made. The images or other third party material in this article are included in the article's Creative Commons licence, unless indicated otherwise in a credit line to the material. If material is not included in the article's Creative Commons licence and your intended use is not permitted by statutory regulation or exceeds the permitted use, you will need to obtain permission directly from the copyright holder. To view a copy of this licence, visit http://creativecommons.org/licenses/by/4.0/.

(C) The Author(s) 2021 